ANALYSEN

Reinhold Grotz

\title{
Der Klimawandel in Australien und die Folgen
}

\section{Australien im Klimasystem des Südpazifik}

\subsection{Das Durchschnittsklima}

Gemessen an seinem Durchschnittsklima ist Australien der trockenste der bewohnten Kontinente der Erde. Dies ist klimatologisch zu erwarten, denn es entspricht der globalen Lage Australiens am südlichen Wendekreis, der durch den Kontinent zieht. Dennoch gibt es Schlagzeilen wie diese: Überschwemmungen in der Region Brisbane im Januar 2011 mit 35 Toten oder Überschwemmungen in und bei Bundaberg im Januar 2013 mit vier Toten.

Um den Widerspruch zu verstehen, ist es hilfreich, einige Grundlagen über die Klimatologie Australiens zu kennen. Im Norden hat Australien Anteil an den wechselfeuchten Tropen mit ausgeprägten Sommerregen zwischen November und März (Zenitalregen). Im Innern des Kontinents herrscht dagegen wegen der Lage des subtropischen Hochdruckgürtels ein weitflächig ausgedehntes Trockenklima, das Halbwüsten schafft. Nur der Süden des Kontinents gehört der warm gemäßigten Klimazone an, wo Winterregen vorherrschen, wenn das subtropische Hochdrucksystem nach Norden rückt und der Südteil des Kontinents in die Westwinddrift mit ihren eingelagerten Tiefs gelangt. Die Insel Tasmanien profitiert fast ganzjährig von ihrer Lage in dieser Westwindzone.

Besonders bevorzugt sind der Südosten und die Ostküste des Kontinents. Diese Bereiche empfangen außer den Winterniederschlägen aus dem Westen auch noch Steigungsregen aus den Südost-Passaten, so dass dort neun und mehr humide (feuchte) Monate verzeichnet werden können. Hier im Süden und entlang der Ostküste liegen die wichtigsten Siedlungs- und Wirtschaftsräume, die sich weit bis in den Norden Queenslands hinein ziehen. 


\subsection{Besondere Ereignisse im Klimageschehen}

Der durchschnittliche physikalische Befund beschreibt jedoch nur die halbe Wahrheit. Seitdem es systematische Aufzeichnungen über Wetterdaten gibt, kennt man auch immer wiederkehrende außergewöhnliche Ereignisse großen Ausmaßes. Sie können sich einerseits in extremen und langen Trockenperioden mit verheerenden Busch- und Waldbränden und andererseits in Intensivniederschlägen mit großflächigen Überschwemmungen äußern. Dadurch entstehen nicht nur enorm hohe wirtschaftliche Schäden, sehr oft sind auch Menschenleben zu beklagen.

Diese Extremereignisse hängen mit Abweichungen von den normalerweise vorherrschenden Windsystemen (Walker-Zirkulation) im Südpazifik zusammen (Hilgers 2012). Der Südost-Passat treibt in der Mehrzahl der Jahre Oberflächenwasser südlich des Äquators von Südamerika aus westwärts. Auf seinem langen Weg erwärmt es sich und sorgt durch zunehmende Verdunstung im indonesisch-australischen Raum für ergiebige Konvektionsniederschläge. Man misst diese Zirkulation durch Luftdruckunterschiede zwischen Tahiti und Darwin. Der Southern Oscillation Index (SOI) ist für das langjährige Mittel des Druckunterschieds zwischen den beiden Wetterstationen auf Null gesetzt (Standardisierung). Länger anhaltende Abweichungen des SOI in den positiven oder negativen Bereich sind Vorboten für Witterungsanomalien.

Aus Gründen, die nicht vollständig erforscht sind, kann es vorkommen, dass sich die Passatwinde abschwächen oder ganz einschlafen, weil die Luftdruckunterschiede deutlich geringer sind als normal und sich ein Tiefdruckgebiet über dem Mittel- oder Ostpazifik bildet. Der Meeresstrom wird schwächer und dreht sich teilweise sogar um. Die hohen Oberflächentemperaturen des Wassers führen bereits im Mittelpazifik zu Konvektionsniederschlägen. Der westpazifische Raum mit Indonesien und Australien bleibt dagegen trocken, weil das nach Osten weggeführte warme Oberflächenwasser durch kühleres Tiefenwasser ersetzt wird. Die Luftdruckverhältnisse haben sich also umgedreht und der SOI ist negativ (kleiner -8). Dies ist ein sogenanntes El Niño-Ereignis mit ausgeprägter Trockenheit im Norden und Osten Australiens (Hilgers 2012).

Wenn nun umgekehrt sehr starke Passatwinde überdurchschnittlich viel warmes Wasser vor die australische Ostküste treiben, kommt es 
dort zu besonders hohen Niederschlägen. Der SOI ist in diesem Fall stark positiv (über +8 ), weil das Druckgefälle in Richtung Australien sehr hoch ist. Dann spricht man von einem La Niña Ereignis. Jetzt kommt es zu lang anhaltenden und auch sehr heftigen Regenfällen, die ausgedehnte Überschwemmungen zur Folge haben können. Im Januar 2011 musste in Queensland und New South Wales eine Fläche so groß wie Deutschland und Frankreich zusammen zum Katastrophengebiet erklärt werden. Erschwerend kam hinzu, dass sich, eingebettet in eine starke Ost-West Strömung, noch ein tropischer Zyklon der höchsten Kategorie 5 (Yasi) eingereiht hatte.

Solche tropical cyclones bringen ebenfalls riesige Regenmengen. Sie sind den amerikanischen hurricanes vergleichbar und entstehen über dem warmen Meer. Ihre Windenergie beziehen sie aus der Freisetzung von Kondensationsenergie bei der Wolkenbildung. Über Land verlieren sie daher bald an Stärke. Aber in Küstennähe entstehen durch hohe Windgeschwindigkeiten (bis über $250 \mathrm{~km} / \mathrm{h}$ ) und extrem hohe Niederschläge innerhalb weniger Stunden (bis 300 mm) Zerstörungen mit katastrophalen Ausmaßen.

Die El Niño-La Niña-Geschehen reichen selten bis nach Western Australia. Die Westküste Australiens scheint jedoch durch eine ähnliche "Luftdruck-Schaukel" im Indischen Ozean bestimmt zu sein. Derzeit wird intensiv über dieses Phänomen geforscht, genaue Erkenntnisse gibt es aber noch nicht.

\subsection{Fazit}

Klimatologisch gesehen gehören also auch diese beschriebenen Wetterextreme zum Normalzustand der Atmosphäre. Bereits in kolonialer Zeit wurde von katastrophalen Wettergeschehen mit ihren Folgen berichtet: ausgedehnte Überflutungen, verheerende Stürme und jahrelange Dürren mit großflächigen Bränden.

Es stellt sich daher die Frage, wie sich unter diesen Umständen die Einflüsse eines erst seit wenigen Jahrzehnten diskutierten globalen Klimawandels herausfiltern lassen. In Australien stritt man sich lange über die Realität eines Klimawandels, was dazu führte, dass Australien erst Ende 2007 nach einem Regierungswechsel das Kyoto-Protokoll unterzeichnete. 
Inzwischen haben jedoch sowohl das Bureau of Meteorology (BoM) als auch die Commonwealth Scientific and Industrial Research Organisation (CSIRO) viele Grundlagen erarbeitet, die einen Klimawandel für Australien wahrscheinlich machen. Einige Fakten seien im Folgenden vorgestellt.

\section{Meßbare Zeichen für einen Klimawandel}

\subsection{Die Zunahme der Durchschnittstemperaturen}

Bei den jährlichen Durchschnittstemperaturen zwischen 1910 und 2012 ergibt sich für die letzten vier Jahrzehnte ein durchschnittlicher Temperaturanstieg von etwa $1^{\circ} \mathrm{C}$. Der Anstieg erfolgte räumlich nicht gleichmäßig. Betroffen sind auch die wichtigsten Siedlungsund Agrarräume im Südosten und entlang der Ostküste (Grotz 2010: 39). Ab 1970 nahm die Zahl der außergewöhnlich heißen Jahre zu, die sich unter die höchsten $5 \%$ der jemals gemessenen Werte einreihen.

Ein Bericht vom BoM und CSIRO sagt für den Zeitraum 2010 bis 2040 in mindestens jedem zweiten Jahr sehr hohe Temperaturen voraus, die $60-80 \%$ der Landesfläche betreffen werden. Der Sommer 2012/13 war der heißeste in der 102-jährigen Geschichte der genauen Wetterbeobachtungen in Australien (Cubby 2013). Am 18. Januar 2013 gab es in Sydneys Observatory Hill mit knapp $46^{\circ} \mathrm{C}$ einen neuen Hitzerekord, am Flughafen und in anderen Stadtteilen wurde die 46-Grad-Marke sogar überschritten. Die Folgen waren Zugausfälle wegen Gleisverwerfungen, Kurzschlüsse wegen durchhängender Strom- und Telefonleitungen, andere technische Defekte, Hunderte von Notaufnahmen in den Krankenhäusern und überdurchschnittlich viele Tote unter der älteren Bevölkerung.

Mit der Hitze steigt auch die Wald- und Buschbrandgefahr. Die katastrophalen Brände in Victoria im Februar 2009 mit 173 Toten sind ohne Vergleich. Vorausgegangen waren Hitzewellen mit über $40^{\circ} \mathrm{C}$. Der Erwärmungstrend auf dem Kontinent hält an, denn im Januar 2013 betrug die Temperaturanomalie für ganz Australien $+2,28^{\circ}$. Dies ist ein neuer Rekord (BoM 2013). 


\subsection{Die Veränderung der jährlichen Niederschläge}

Parallel zu den Temperaturerhöhungen beobachtet man innerhalb der stark oszillierenden Jahresniederschlagswerte seit den 1970er Jahren, dass die wichtigsten Agrargebiete im Südosten und im Südwesten tendenziell trockener werden. Dieser Trend ist eindeutig, wenn man kurzfristige Schwankungen durch die Angabe von 10-Jahreswerten glättet (Grotz 2010: 38). Die im Norden und im Zentrum von Western Australia feuchter gewordenen Landesteile sind für den wirtschaftenden Menschen kaum von Bedeutung.

Treffen in einer Region geringere Niederschläge mit höheren Temperaturen zusammen, können bei längerer Dauer Dürren (droughts) auftreten. Diese Kombination verursachte und verschlimmerte die droughts im letzten Jahrzehnt vor allem im Murray-Darling Becken. Dieses Flusssystem entwässert ca. 1 Mio. km² (etwa dreimal die Fläche Deutschlands). Hier werden fast $40 \%$ aller Agrarwerte erzeugt, etwa die Hälfte der Getreideernte kommt von hier, ebenso weiden hier $45 \%$ aller Schafe und die größten Mengen Obst und Gemüse sowie viele andere Produkte werden hier geerntet.

Entlang der Flüsse wurden Dämme, Kanäle und andere Bewässerungseinrichtungen geschaffen, aber das bewässerbare Land macht nur etwa $2 \%$ des Agrarlandes aus. Es ist jedoch für die ertragreichen Kulturen wie Reis, Baumwolle, Obst, Gemüse und Wein unbedingt nötig.

Die Niederschlagsdefizite im letzten Jahrzehnt ließen die Zuflüsse zum Murray drastisch zurückgehen (Grotz 2010: 41). Der Fluss erreichte viele Jahre lang nicht mehr das Meer. Dafür war zudem die um $15 \%$ erhöhte Verdunstung durch den Temperaturanstieg verantwortlich. Auch die Speicher des Snowy Mountains Hydro-Electric Scheme waren weitgehend geleert. Um die vertraglich vereinbarte Stromversorgung für Victoria dennoch zu sichern, installierte das Hydro-Electric Scheme Gasturbinen mit einer Leistung von 620 MW. Die Wasserversorgung von Adelaide war ebenfalls in Gefahr und die nach der Ramsar Convention als schützenswert eingestuften Habitate in Feuchtgebieten für endemische Pflanzen- und Tierarten trockneten aus (z.B. river red gum, black box tree, Murray Karpfen). 


\subsection{Extremereignisse}

Listet man die Folgen der außergewöhnlichen Wetterereignisse der letzten Jahre mit ihren Schäden - soweit sie überhaupt feststellbar waren - auf, dann ergibt sich eine eindrucksvolle Liste (Tabelle 1).

Tabelle 1: Katastrophale Wetterereignisse

\begin{tabular}{|l|l|l|l|l|l|}
\hline Zeit & Region & Ursache & Tote & $\begin{array}{l}\text { Schäden } \\
\text { Mrd. AUD }\end{array}$ & $\begin{array}{l}\text { Bemer- } \\
\text { kungen }\end{array}$ \\
\hline $\begin{array}{l}\text { April } \\
1999\end{array}$ & Sydney & Hagel & $?$ & 4,3 & $\begin{array}{l}\text { nur Ver- } \\
\text { sicherungen } \\
\text { (Preise } \\
\text { 2011) }\end{array}$ \\
\hline $\begin{array}{l}2002- \\
2010\end{array}$ & $\begin{array}{l}\text { Südost- } \\
\text { Australien }\end{array}$ & Dürre & $?$ & 4,5 & $\begin{array}{l}\text { nur Staats- } \\
\text { ausgaben }\end{array}$ \\
\hline $\begin{array}{l}\text { Febr. } \\
2009\end{array}$ & Victoria & Feuer & 173 & 4,4 & \\
\hline $\begin{array}{l}\text { Jan. } \\
2011\end{array}$ & $\begin{array}{l}\text { Ost- } \\
\text { Queensland }\end{array}$ & $\begin{array}{l}\text { Überschwem- } \\
\text { mungen }\end{array}$ & 35 & 4,7 & \\
\hline $\begin{array}{l}\text { Jan. } \\
2013\end{array}$ & $\begin{array}{l}\text { Nordost- } \\
\text { Queensland }\end{array}$ & $\begin{array}{l}\text { Überschwem- } \\
\text { mungen }\end{array}$ & 4 & 2,4 & \\
\hline
\end{tabular}

Quelle: Eigene Zusammenstellung

Die mittleren und kleineren Katastrophen mit weniger als 2 Mrd. AUD Schaden (etwa 1,5 Mrd. $€$ ) sind hier nicht aufgeführt. Produktions-ausfälle in der Landwirtschaft und im Bergbau sind ebenfalls nicht berücksichtigt. Beispielsweise schmälerten die Wirkungen des Cyclones Yasi mit anschließenden Überschwemmungen in Queensland im Januar 2011 die gesamte Volkswirtschaft um ein halbes Prozent (etwa 65 Mrd. AUD), weil Kohletagebaue monatelang unter Wasser standen, Straßen und Bahnlinien weggeschwemmt wurden und beispielsweise $95 \%$ der australischen Bananenfelder vernichtet waren. Paradoxerweise rechnet die volkswirtschaftliche Gesamtrechnung die Leistungen für die Schadensbehebung auf der positiven Seite. In Wirklichkeit waren die volkswirtschaftlichen Einbußen viel höher als ein halbes Prozent. Schätzungen ergaben 100 Mrd. AUD (ca. 75 Mrd. $€$ ). 
Zum Vergleich verursachte das Elbehochwasser 2002 materielle Schäden einschließlich Katastrophenbekämpfung und Produktionsausfällen in Höhe von ca. 11,4 Mrd. $€$ (etwa 15,2 Mrd. AUD). Damals hatten Sachsen 21 und Tschechien 17 Todesopfer zu beklagen. Möglicherweise übertreffen die Schäden des Hochwassers von 2013 die früheren Werte.

Naturbedingte Schäden kleineren oder mittleren Ausmaßes ereignen sich in Australien häufig, ohne dass die Weltöffentlichkeit große Notiz davon nimmt. Im Februar 2013 erzwang der Cyclone Rusty (Kategorie 3) im Pilbara-Gebiet einen kurzen Stopp der Eisenerzförderung, die Erzschiffe wurden zu ihrer Sicherheit aus den Häfen Port Hedland und Umgebung auf den Indischen Ozean hinaus gesandt. Die Erzverladung ruhte drei Tage lang. Obwohl sich Rusty an Land rasch abschwächte, war die Stromversorgung teilweise unterbrochen und Hunderte Menschen mussten in Schutzräume fliehen. Kurz zuvor hatten die Ausläufer des Cyclone Oswald in Queensland Schäden in Höhe von 2,4 Mrd. AUD (1,8 Mrd. $€$ ) verursacht.

Gegen solche Naturereignisse können sich Menschen nur mit einem sehr hohen Aufwand schützen. Versicherungen helfen nur bedingt, denn die Prämien steigen mit jeder Katastrophe stark an. Sie wurden von September 2011 bis September 2012 um 16\% angehoben. Ein Beispiel: Für Häuser mit einem Überschwemmungsrisiko bis maximal einem Schadensfall in 50 Jahren und einem Versicherungswert von 390000 AUD fordert Allianz eine Jahresprämie von 4700 AUD (etwa $3500 €$ ) in New South Wales und von 8000 AUD (ca. 6000 $€$ ) in Queensland. Allianz berichtet, dass $98 \%$ der potentiellen Kunden dieses Angebot ablehnen. Man hofft, dass es schon gut gehen wird.

Aufgrund der vielen Schadensfälle in den letzten Jahren wurde Australien zu Beginn 2013 im Markt der international tätigen Rückversicherer zurück gestuft. Australische Versicherungen müssen nun für ihre eigene Rückversicherung höhere Prämien bezahlen, die sie natürlich an ihre Kunden weitergeben.

Weil die Schäden an der öffentlichen Infrastruktur oftmals die finanziellen Möglichkeiten staatlicher Institutionen übersteigen, gab es 2011 für nicht geschädigte Steuerzahler eine Sondersteuer für Besserverdiener über 50000 AUD Jahreseinkommen (ca. $38000 €$ ) in 
Höhe von 0,5\% des Einkommens. So wurden etwa 1,8 Mrd. AUD erhoben.

Nach der Vielzahl der geschilderten Extremereignisse mit Hitzewellen, Dürren, Zyklonen und Überschwemmungen mit ihren Folgen liegt es nahe, auf einen Klimawandel zu schließen. Die 2012 veröffentlichten Ergebnisse des IPCC (Intergovernmental Panel on Climate Change) stützen diese Ansicht aber nur zum Teil. Wie bereits erwähnt, gehört wild weather zur Natur des Kontinents. Nach den Erkenntnissen des IPCC werde es in Zukunft zwar quantitativ nicht mehr Extremereignisse geben, jedoch werde ihre Intensität zunehmen.

Die Temperaturerhöhung verschärft die Dürre- und Brandgefahr an Land. Da die höheren Temperaturen ebenso über den Meeren gemessen werden, führen sie dort zu einer stärkeren Verdunstung. Trotz der beobachteten insgesamt geringeren Landniederschläge kommt es je nach Berechnungsmethode zu einer um 5,9 bis 7,7\% höheren Niederschlagsintensität. Dies sowie eine steigende Besiedlungsdichte in gefährdeten Gebieten und mehr Investitionen durch größeren Wohlstand verursachen die enorm hohen Schadenssummen. Wenn sich die Atmosphäre bis 2030 um ein weiteres Grad erwärmt - wovon auszugehen ist - stehen Australien viel größere Katastrophen bevor als die bisher erlebten (CSIRO 2013).

Dessen ist man sich inzwischen in Australien bewußt und man beginnt, sich für die neuen Entwicklungen zu wappnen (PMSEIC 2007). Seit einigen Jahren gibt es einen für die Klimaänderung zuständigen Minister in Canberra (heutige Bezeichnung: Minister for Climate Change, Industry and Innovation). Es werden Forschungen initiiert, finanziert und koordiniert. Im Jahre 2006 wurde zusammen mit den Regierungen der Bundesstaaten beschlossen, einen Plan zur Anpassung an den Klimawandel zu entwickeln, an dem auf verschiedenen Teilgebieten laufend gearbeitet wird (Department of Climate Change 2010, 2011a). Im Folgenden sollen einige Themenbereiche mit ihren Einflüssen aus dem Klimawandel angesprochen werden. 


\section{Folgen des Klimawandels und mögliche Anpassungen}

\subsection{Biosphäre und Gesundheit}

Die aus globaler Sicht größte Gefährdung betrifft das Great Barrier Reef. Das über $2000 \mathrm{~km}$ lange Riff vor der Küste Nordost-Queenslands, das als eines der ersten zum Weltnaturerbe erklärt wurde, ist von 400 Arten von Korallen, 1500 Fischarten und 240 Vogelarten bevölkert. Dieses Ökosystem gerät durch steigende Wassertemperaturen, die zunehmende Versauerung des Wassers durch $\mathrm{CO}_{2}$, also Kohlensäure, sowie durch Schlamm- und chemische Einträge vom Festland her in Gefahr. Gestiegene Temperaturen und saures Wasser erschweren die Abscheidung von Kalk durch die winzigen Korallenpolypen und auch die wichtigen Symbiosen mit anderen Organismen, die zudem für die oft leuchtenden Farben verantwortlich sind, leiden. Die Riffe bleichen aus und schließlich sterben sie ab. Die abfließenden Hochwässer vom Festland bringen aus den Agrargebieten nicht nur Schlamm, sondern auch Düngemittel und Pestizide mit sich. Durch die Düngung des Meeres überwuchern Großalgen die Korallenstöcke und bringen sie zum Absterben.

Die Temperaturerhöhung des Wassers begünstigt die Ausbreitung von tropischen und subtropischen Tieren nach Süden, u. a. sind die für manche Badegäste sogar tödlichen Nesselquallen auf dem Weg nach Süden. Auch an Land breiten sich tropische Tiere und Pflanzen südwärts aus. Andererseits finden Tier- und Pflanzenarten der gemäßigten Zone keine neuen Ausweichräume mehr. Insbesondere Biotope in den Snowy Mountains sind vom Aussterben bedroht.

Auf eine auch für Touristen unangenehme Folge des Klimawandels sei hingewiesen. Tropische Erkrankungen fassen bereits auf dem Fünften Kontinent Fuß. Zwar gibt es noch keine endemischen Malaria-Fälle oder Gelbfieber, aber das Dengue-Fieber, eine Viruserkrankung mit hohem Fieber, heftigen Kopf- und Gliederschmerzen und inneren Blutungen, ist nach Queensland eingewandert. Es wird durch Stechmücken übertragen und kommt entlang fast der gesamten Queensland-Küste vor. Zwischen 2011 und 2012 verdoppelte sich die Zahl der Erkrankungen. Aber noch ist die Fallzahl etwa im Vergleich zu Brasilien gering.

Wie zu erwarten war, verbreiten sich jetzt auch tropische Tierkrankheiten schneller und weiter. Beispielsweise ist das Tick-Fieber bei 
Rindern an der Ostküste rasch im Vormarsch (Übertragung durch Zecken) und hat bereits New South Wales erreicht. 20\% der erkrankten Tiere bei europäischen und nordamerikanischen Rassen verenden. Die Einkreuzung von Erbgut aus Brahman-Rassen soll die Resistenz erhöhen.

\subsection{Landwirtschaft}

Naturgemäß ist dieser Wirtschaftszweig am meisten von einem Klimawandel betroffen. Wenn die Veränderungen bei Temperaturen und Niederschlägen nicht zu rasch ablaufen, kann sich eine auf Wissenschaft basierte Landwirtschaft auf die neuen Verhältnisse einstellen. Dazu müssen allerdings die Akteure das nötige Wissen erwerben. Tatsächlich besitzt in Australien ein Drittel der in der Landwirtschaft Tätigen eine Hochschul- oder andere Spezialausbildung. Daher sollte die Verbreitung und Anwendung neuen Wissens kein großes Problem sein. Wenn jedoch zu den schleichenden Veränderungen Extremereignisse, z.B. mehrjährige Dürren, Brände, tropische Zyklone und andere wolkenbruchartige Regenfälle - und das mit steigender Intensität - hinzu kommen, helfen wissenschaftliche Erkenntnisse einer modernen Farmbewirtschaftung kaum weiter. Die Farmer müssen zukünftig mit noch höheren und unkalkulierbaren Risiken wirtschaften.

Gesamtwirtschaftlich gesehen ist die Landwirtschaft in Australien kein wichtiger Wirtschaftszweig mehr und ihre Bedeutung nimmt weiter ab. Die Beiträge zum Bruttoinlandsprodukt (BIP) wie zur Beschäftigung liegen zwischen 2,5 und 3\%. Obwohl die Arbeits- und Kapitalproduktivität allgemein hoch sind und daher rund $60 \%$ des erzeugten Wertes exportiert werden können (Getreide, Ölsaaten, Fleisch, Baumwolle, Wolle), machen diese Exporte nur noch $10-11 \%$ der gesamten Exportleistung aus (2011/12). Etwa 60\% der Ausfuhren stammen aus dem Bergbau.

Die Landwirtschaft durchläuft nicht zuletzt wegen der Auswirkungen der langen Dürre im letzten Jahrzehnt einen raschen Wandel. 
Tabelle 2: Merkmale der Landwirtschaft in Australien und ihre Veränderungen

\begin{tabular}{|l|l|l|l|l|}
\hline & 2001 & $\mathbf{2 0 1 1}$ & \multicolumn{2}{|c|}{ Veränderungen } \\
\cline { 3 - 5 } & & & absolut & in \% \\
\hline Agrarbetriebe in 1000 & 141 & 136 & -5 & $-3,6$ \\
\hline Nutzfläche in Mio. ha & 456 & 410 & -46 & $-10,1$ \\
\hline $\begin{array}{l}\text { davon Ackerfläche, Obst } \\
\text { usw. in Mio. ha }\end{array}$ & 25 & 32 & +7 & $+30,8$ \\
\hline $\begin{array}{l}\text { Bewässerungsfläche } \\
\text { in Mio. ha }\end{array}$ & 2,6 & 2,0 & $-0,6$ & $-24,5$ \\
\hline
\end{tabular}

Quelle: Australian Bureau of Statistics, No. 7106.0, 2012 (verändert; Zahlen gerundet)

Während der letzten Dekade erlebte Australien wegen der lang anhaltenden Dürre in einigen Jahren fast völlige Ernteausfälle in wichtigen Anbaugebieten. Nur weil in anderen Regionen etwas bessere Bedingungen herrschten, verzeichneten die Statistiken noch Erntemengen, wenn auch geringere.

Beispielhaft soll dies an den Anbauflächen und den Ernteerträgen von Weizen ab dem Wirtschaftsjahr 1999-2000 gezeigt werden.

Abbildung 1: Anbauflächen und Erntemengen von Weizen



Quelle: Australian Bureau of Statistics 
In guten Jahren können 25 Mio.t Weizen geerntet werden, von denen 15-20 Mio.t auf den Weltmarkt gelangen. Die Mengen im relativ trockenen Jahr 2003-04 waren nur deshalb so gut, weil es in Western Australia und Victoria Rekordernten gab, während die Farmer in New South Wales und im Süden von Queensland Missernten verzeichneten bzw. wegen der Trockenheit gar nicht aussäen konnten. In den beiden Wirtschaftsjahren 2006-07 und 2007-08 waren die durchschnittlichen Hektarerträge nur etwa halb so hoch wie in den Jahren zuvor und danach.

Trotz der immer wiederkehrenden Ernteeinbrüche lässt sich eine erstaunliche Beobachtung machen. Vergleicht man die ha-Erträge ganzer Jahrzehnte miteinander, so dass einzelne El Niño-Jahre und andere Wetterkatastrophen nicht so sehr hervortreten, dann lassen sich von Jahrzehnt zu Jahrzehnt trotz höherer Temperaturen und niedrigerer Niederschläge dennoch höhere Ernten pro Hektar errechnen. Lagen sie in den 1960er Jahren erst bei $12 \mathrm{dt} / \mathrm{ha}(1 \mathrm{dt}=100 \mathrm{~kg})$, stiegen sie bis in die 1990er Jahre auf durchschnittlich 16,5dt/ha. Selbst im letzten Dürrejahrzehnt sank der Mittelwert nur wenig auf 15,9 . In den Erntejahren 2009-10 bis 2011-12 ergaben sich nochmals Steigerungen bis nahe 20dt/ha. Dies bedeutet: Trotz erschwerter Umweltbedingungen erzielten die Weizenfarmer eine Steigerung der Flächenerträge von über $50 \%$. Das sind Erfolge von Neuzüchtungen, besseren Fruchtfolgen, Feuchtigkeit konservierender Bodenbearbeitung sowie einer gezielten Düngung.

Bisher ist es den Farmern mit Hilfe der Forschung offensichtlich gelungen, in der Summe die negativen Effekte des Klimawandels aufzufangen. Daher empfiehlt der CSIRO den Farmern als Anpassungsstrategie keinen radikalen Wandel ihrer Wirtschaftsweise, sondern konsequent best practice-Methoden anzuwenden. Dies erfordert eine sehr flexible Betriebsführung, die zunehmend vom jeweiligen Wetter- und Klimarisiko bestimmt sein wird (CSIRO 2011). Alle Risiken lassen sich nicht berechnen oder durch Vorsorge ausschalten. Sie können einzelne Farmer oder ganze Regionen hart bis zur Gefährdung ihrer Existenzgrundlagen treffen (Brände, Dürren, Stürme und Überschwemmungen).

Auf Dauer werden kleine, schrittweise Anpassungen für manche Betriebe nicht ausreichen. Auch der CSIRO sieht die Notwendigkeit, dass ökonomisch schwache Weizenfarmen aufgeben. Man schätzt bis 
2030 einen Rückgang der gesamten Weizenproduktion um 8-10\%. Eine Aufgabe von Grenzertragsbetrieben ist schon deshalb erforderlich, weil die Bodendegradation bei stark geschädigter oder fehlender Vegetation riesige Ausmaße annimmt. Dies betrifft sowohl die Windausblasung von fruchtbarem Oberboden als auch die Rinnenerosion bei Starkregen. Ein Rückzug der Landwirtschaft aus Problemgebieten ist daher sehr erwünscht, denn bereits heute verursachen ökologische Schäden enorm hohe Landwertverluste und Ernteausfälle, die sich unter den Bedingungen des Klimawandels nochmals steigern.

Abschließend noch einige Bemerkungen zur Bewässerung. Sie kann in einem wärmer werdenden Klima die Erträge sichern. Im MurrayDarling Becken, wo etwa zwei Fünftel der Agrarproduktion herkommen, sind Felder mit Reis, Baumwolle, Wein, Obst, Gemüse und selbst Fettweiden für Milch- und Mastvieh bewässert. Bei abnehmendem Wasserangebot sind zukünftig zwangsweise hohe Produktionseinbußen zu erwarten.

Abbildung 2: Anbauflächen und Erntemengen von Baumwolle

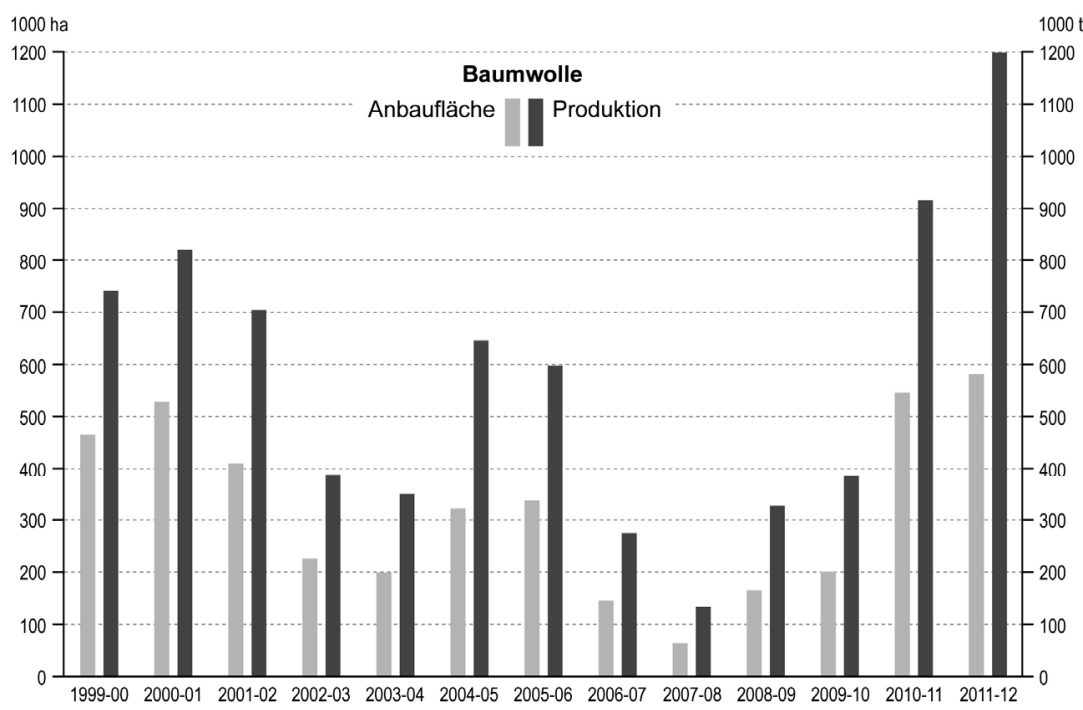

Quelle: Australian Bureau of Statistics 
Abbildung 2 zeigt die Schwankungen bei den Anbauflächen und der Produktion von Baumwolle während des letzten Jahrzehnts. Baumwolle ist als cash crop für die Farmer sehr wichtig, sie wird fast völlig exportiert und erbringt gute Preise. Daher ist Baumwolle statistisch eine der wichtigsten Anbaufrüchte. Obwohl die ha-Erträge in 40 Jahren fast verdoppelt werden konnten, droht dem Anbau das Aus, wenn kein Bewässerungswasser mehr zur Verfügung steht.

Der Kampf um die Verteilung des Wassers im Murray-Darling Basin zwischen den Bundesstaaten und verschiedenen Nutzungsarten ist längst entbrannt. Regierungen kaufen mit Milliarden-Programmen Wasserrechte von Farmern auf. Doch immer wieder regt sich Widerstand, wenn Farmer nicht nur zur Sicherung der Trinkwasserversorgung von Städten, sondern auch zugunsten der Wasserqualität von Flüssen oder zur Lebenserhaltung von wertvollen Feuchtbiotopen auf Wasserrechte verzichten sollen. Fisch- und Baumsterben, giftige Algenblüten im Wasser und das Verschwinden ganzer Ökosysteme sind zwar Nachrichten wert, doch wirtschaftliche Eigeninteressen verhindern oft ein notwendiges Handeln. Nach vielen Jahren intensiver Forschungen und Verhandlungen mit Beteiligten ist es der Murray-Darling Basin Authority bis heute nicht gelungen, ein allgemein akzeptiertes neues Gleichgewicht der Wasserverteilung zu finden. Andererseits gehen immer noch viele Bewässerungsmethoden sehr verschwenderisch mit dem knappen Gut Wasser um, was an manchen Stellen zu sehr negativen Bodenversalzungen führt.

\subsection{Siedlungen}

Die lange Niederschlagsarmut im letzten Jahrzehnt brachte für die fünf Millionenstädte - Sydney, Melbourne, Brisbane, Perth und Adelaide - in der Wasserversorgung große Engpässe. Alle diese Städte verfügen über große Wasserspeicher in Form von Stauseen, die aber nicht mehr den Bedarf decken konnten. Überall musste ein gestuftes System der Wasserrationierung eingeführt werden. Zusätzlich mussten innerhalb weniger Jahre Meerwasser-Entsalzungsanlagen geplant und gebaut werden, die je nach Stadt $15-50 \%$ des Bedarfs decken können. In Brisbane sieht man im Notfall sogar die Aufbereitung von Schmutzwasser als Trinkwasser vor. Um generell den Wasserverbrauch in den Haushalten zu senken, machen öffentliche Kampagnen und Förderanreize auf Wasser sparende Installationen aufmerksam. 
Dazu gehört auch die Einrichtung von Regenwassertanks, die wenigstens teilweise den Bedarf für Rasen und Garten decken sollen.

Die Wasserfrage ist in hoch entwickelten Staaten weniger eine Existenz- als eine Kostenfrage. Die fünf bisher gebauten großen Meerwasser-Entsalzungsanlagen kosteten etwa $10 \mathrm{Mrd}$. AUD (7,5 Mrd. $€)$. Da sie laufend hohe Energiemengen benötigen, klingt es paradox, dass erst ein kleiner Teil dieser Energie aus erneuerbaren Quellen stammt.

Größere Probleme werden vor allem die großen Städte mit dem Hitzestress bekommen. Australienweit war der Sommer 2012-13 der heißeste seit 1910, wobei sechs der zehn heißesten Sommer erst innerhalb der letzten zehn Jahre gemessen wurden. In den Städten ist die Hitzeentwicklung durch die wenig reflektierenden, Wärme speichernden Baumassen besonders hoch und auch die Nächte sind immer öfter wärmer als $30^{\circ}$. Der Chefmeteorologe des australischen Wetterdienstes sagte voraus, dass Sommer mit Tagestemperaturen über $40^{\circ} \mathrm{C}$ in etwa 40 Jahren "normal" wären (Hannam 2013).

Solange die Wohnhäuser - traditionell Einfamilienhäuser - auf großen Grundstücken mit hohen Schattenbäumen stehen, waren für die Bewohner auch hohe Temperaturen bisher noch erträglich. In den zunehmend verdichteten Stadtzentren mit Büro- und Wohnhochhäusern entwickeln sich Hitzepole, die das Leben ohne Klimatisierung unmöglich machen. Die kühlende Wirkung von Seewinden endet nach wenigen Kilometern landeinwärts; sie hat in den dicht bebauten Innenstädten ohnehin nur wenig Wirkung. Weniger natürliche Kühlung gibt es auch in den Neubaugebieten der Vororte. Dort stehen die neuen, mittlerweile über $250 \mathrm{~m}^{2}$ Wohnfläche großen Einfamilienhäuser auf kleineren Grundstücken als früher mit zwangsweise kleineren Grünflächen und nur wenigen oder gar keinen Schattenbäumen.

Eine sehr große Herausforderung wird die nachträgliche bessere Thermoisolierung und Klimatisierung von Wohngebäuden sein, die älter als 20-30 Jahre alt sind. Auf Hausbesitzer kommen hohe Investitionen zu. Die Elektrizität für die Kühlung sowie für die Heizung im Winter kommt bisher weit überwiegend aus der Kohle. Die KohleLobby - Minenbetreiber und Bergarbeiter - konnten bis vor wenigen Jahren erfolgreich den Ausbau alternativer Stromquellen verhindern. 2012 stammten erst 13\% der Elektrizität aus erneuerbaren Energien, überwiegend aus Wasserkraft und Biomasse (im Wesentlichen 
Verbrennung von Zuckerrohr-Rückständen) - und das in einem Land mit riesigen Potentialen der Solar- und Windenergieerzeugung. Auch bei der Grün- und Freiraumplanung in Städten muss wegen der jetzt höheren Baudichten und kleineren Gärten umgedacht werden.

Städter werden unter dem Klimawandel am meisten zu leiden haben. Von den fast 23 Millionen Australiern leben bereits $60 \%$ in den fünf Millionenstädten. Zählt man die wenigen weiteren großstädtischen Agglomerationen hinzu (Canberra, Newcastle, Gold Coast usw.), dann kommt man bereits auf über 70\% der Gesamtbevölkerung. Dabei wächst die Einwohnerzahl nach den Maßstäben hoch entwickelter Staaten rasch weiter an. In den letzten Jahren waren es $1,5-1,7 \%$ pro Jahr. Zu einer leicht gestiegenen Geburtenrate kommen wieder deutlich gestiegene Einwanderungszahlen. 85\% der Einwanderer lassen sich in einer der wenigen Großstädte nieder. Dort tragen sie auch zum urban sprawl bei, denn der Wachstumsdruck für Wohnungsbau und neue Infrastrukturen ist enorm hoch (Searle und Braun 2012).

Auf ein spezielles Problem sei besonders hingewiesen. Aus Unachtsamkeit, Unwissenheit oder auf wirtschaftlichen Druck hin sind große durch Hochwässer gefährdete Flächen entlang von Flüssen mit Gebäuden und Verkehrsinfrastruktur bebaut worden. Da über Jahrzehnte keine Fluten zu verzeichnen waren, glaubte man sich dort sicher. Die zunehmende Intensität extremer Wetterereignisse strafte jedoch solche optimistischen Annahmen in den letzten Jahren Lügen.

Die bereits seit ihrer Frühzeit immer wieder von Hochwassern heimgesuchte Stadt Grafton an der Nordküste von New South Wales schützte sich früh durch Dämme und Hochwassermauern. So überstand die Stadt im Januar 2013 ein 8-Meter-Hochwasser nahezu unbeschadet. Andere Städte bauten ohne Schutzmaßnahmen nahe an ihre Flüsse und mussten kostspielige Schäden und sogar Todesfälle in Kauf nehmen. Ein Teil der Schäden geht auf Planungsversagen zurück, daher sind im Hochwasserschutz selbst im Binnenland noch riesige Aufgaben zu bewältigen.

Die Vorsorge für die Brandgefahr bei langen und intensiven Trockenperioden benötigt ebenfalls deutlich mehr Aufmerksamkeit. Die Brandbekämpfung außerhalb größerer Städte basiert bis heute überwiegend auf freiwilligen Diensten. Künftig werden noch mehr Professionalität und eine noch bessere Ausrüstung erforderlich sein. Bilder 
eines Hubschraubers, der Löschwasser aus einem swimming pool zieht, sind zwar spektakulär, wirken aber etwas hilflos angesichts großflächiger Brände.

\subsection{Küstenschutz}

Eines der größten Probleme, das bis heute kaum wahrgenommen wird, betrifft den Küstenschutz. Durch die global höheren Durchschnittstemperaturen kommt es zur Ausdehnung des Wassers und die Eismassen an den Polkappen und auf Grönland schmelzen. Der Meeresspiegel steigt. Für Australien wurden zwischen 1990 und 2010 pro Jahr Werte im einstelligen Millimeterbereich gemessen (Department of Climate Change 2011b). Dabei fallen je nach Seewassertemperatur, Meeresströmungen, Ebbe- und Flutverhältnissen die Anstiege entlang der Küste verschieden hoch aus. Die Gefahrenlage an den Küsten wird sich deutlich erhöhen. Andererseits ist der Zug der Menschen zum Meer ungebrochen. Die neuesten IPCC-Ergebnisse halten einen durchschnittlichen Anstieg der Randmeere um Australien bis zum Ende des Jahrhunderts in Höhe von 1-1,5 m für sehr wahrscheinlich.

Für Australien wird derzeit eine Risikoabschätzung vorgenommen, der ein Meeresspiegelanstieg von $110 \mathrm{~cm}$ zugrunde liegt. Hinzu kommen noch die Einflüsse von Springtiden und Sturmfluten. Ziel dieser Risikobewertung ist es, Planungsentscheidungen auf allen Planungsebenen bis herab zu den Kommunen zu beeinflussen und Vorsorgemaßnahmen einzuleiten.

Die entwickelten Überflutungsmodelle sind zwar noch vorläufig, die ersten Ergebnisse sind jedoch teilweise erschreckend. Bedeutende Küstenstädte, wie Adelaide oder Brisbane, könnten ohne Schutzmaßnahmen großflächig überflutet werden.

Das Ministerium stellte die Anzahl und den Wert der gefährdeten Wohn- und Gewerbegebäude sowie der Straßen und Bahnlinien zusammen. Wegen der Unsicherheiten wurden eine obere und eine untere Grenze der Gefährdung festgelegt. Selbst die optimistische untere Schätzung ergibt ein Risiko für Bauwerte nach dem Preisstand von 2008 in Höhe von mindestens 226 Mrd. AUD (170 Mrd. $€$ ). Die Bundesstaaten Queensland und South Australia sind am meisten betroffen (Department of Climate Change 2011b). Wie real solche Ge- 
fährdungen vom Meer her heute bereits sind, lässt sich aus Fotos jüngerer Sturmfluten erahnen.

\section{Fazit und Ausblick}

In Australien beginnen die letzten Skeptiker, den Klimawandel auch auf ihrem Kontinent als Realität anzuerkennen. Der Direktor des Climate Change Institute an der Australian National University berechnete eine statistische Chance von 1:500, dass das Wettergeschehen der letzten Jahre noch innerhalb der bisherigen natürlichen Variationsbreite liegt. Zur allgemeinen Akzeptanz des Klimawandels trug auch der heiße Sommer 2012-13 bei. Von den 21 Tagen mit Rekordhitzen von über $39^{\circ} \mathrm{C}$ kontinentaler Durchschnittstemperatur, die in 102 Jahren gemessen wurden, lagen acht im Jahre 2013 (Cubby 2013). Auch die Regenextreme stellten immer neue Rekorde auf. Sie trugen aber mehr zur Landdegradation bei als zum Pflanzenwachstum.

Eine interdisziplinäre Arbeitsgruppe von Wissenschaftlern machte 2010 für das Weltwirtschaftsforum in Davos den Versuch, die für Australien zu erwartenden Schäden aus Umweltrisiken für das laufende Jahrzehnt bis 2020 zusammenzustellen (Grotz 2012: 77). Dabei ergaben sich sehr hohe Geldsummen hauptsächlich aus Extremereignissen. Der für Flussüberschwemmungen angegebene Schätzwert wurde bereits Anfang 2013 überschritten. Dabei lassen sich dauerhafte Schäden aus dem Verlust von Biodiversität und Bodenzerstörung monetär schlecht beziffern. Ursächlich verhindernde Maßnahmen sind den Australiern - außer einer drastischen Reduzierung des Ausstoßes von Treibhausgasen - kaum möglich. Australier können eigentlich nur die schädlichen Auswirkungen abmildern.

Auch das Bureau of Meteorology musste sich dem Klimawandel anpassen. Im Mai 2013 wurde ein neues Wettervorhersagemodell eingeführt, in dem die Erfahrungen aus dem geschichtlichen Wettergeschehen ein deutlich geringeres Gewicht erhalten als bisher. Mehr aktuelle Messdaten, z. B. aus Satelliten und Messbojen in den Meeren um Australien sowie neue Modelle sollen bessere Vorhersagen auch für Extremereignisse ermöglichen.

Zusammenfassend lässt sich sagen, dass Australien durch den Klimawandel in viel stärkerem Maße betroffen ist als etwa Mitteleuropa. Australien kann sich als hoch entwickeltes und vergleichsweise 
reiches Land - wenn auch nur in begrenztem Maße - vor den negativen Folgen des Klimawandels schützen und mit den Schäden fertig werden. Doch abschließend sei die Frage gestellt: Welche Möglichkeiten des Schutzes und der Schadensbewältigung besitzen andere, weniger hoch entwickelte Staaten im südpazifischen Raum?

\section{Bibliografie}

BoM, Bureau of Meteorology, Ed. 2013. Special Climate Statement 43. "Extreme Heat in January 2013". www.bom.gov.au/climate/ current/statements/scs43e.pdf.

CSIRO, Ed., 2011. Climate Change: Science and Solutions for Australia. Canberra: CSIRO PUBLISHING. 85-94.

CSIRO, Ed., 2013. Recent Trends in and Preparedness for Extreme Weather Events. CSIRO Submission 12/471 Senate Standing Committee on Environment and Communications. Canberra: CSIRO.

Cubby, Ben, 2013. "Trends create angry summer". Sydney Morning Herald: March 4. www.smh.com.au/environment/weather/ trends-create-angry-summer-20130303-2fefl.html.

Department of Climate Change, Ed., 2010. Adapting to Climate Change in Australia. Canberra: Commonwealth of Australia.

Department of Climate Change, Ed., 2011a. Climate Change Potential Impacts and Costs Fact Sheets. www.climatechange.gov. au/climate-change/impacts/national-impacts/act-impacts.aspx.

Department of Climate Change, Ed., 2011b. Climate Change Risks to Coastal Buildings and Infrastructure. Canberra: Commonwealth of Australia.

Grotz, Reinhold, 2010. "Auswirkungen der Trockenheit in Australien 2002-2009", in: Altendorf, Ulrike und Liesel Hermes, Hrsg., Australien - Facetten eines Kontinents. Tübingen: Stauffenburg Verlag (KOALAS 9), 33-61.

Grotz, Reinhold, 2012. "Populate and Perish? Folgen eines hohen Bevölkerungswachstums in Australien", in: von Holleuffer, Henriette und Adi Wimmer, Hrsg., Australien - Realität - Klischee Vision. Trier: Wissenschaftlicher Verlag Trier (KOALAS 10), 6984. 
Hannam, Peter, 2013. "Summer a Scorcher but City Set for a Soggy Autumn". Sydney Morning Herald: March 2. www.smh.com.au/ data-point/summer-a-scorcher-but-city-set-for-a-soggy-autumn20130301-2fbp6.html.

Hilgers, Alexandra, 2012. "Dürren, Buschbrände und Überschwemmungen in Australien". Geographische Rundschau, 64 (10), 410.

PMSEIC, Independent Working Group, 2007. Climate Change in Australia: Regional Impacts and Adaptation - Managing the Risk for Australia. Canberra.

Searle, Glen und Boris Braun, 2012. "Neue Trends in Stadtplanung und -entwicklung australischer Großstädte". Geographische Rundschau, 64 (10), 12-19. 